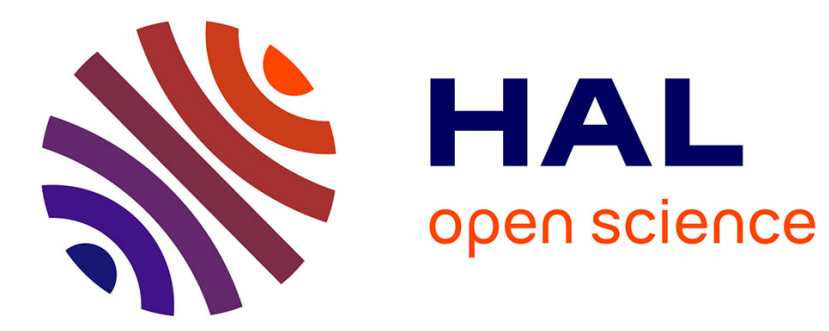

\title{
Physical gels and biopolymers
}

M. Rinaudo

\section{- To cite this version:}

M. Rinaudo. Physical gels and biopolymers. Physics of Finely Divided Matter, Springer Verlag, pp.16-20, 1985. hal-00309370

\section{HAL Id: hal-00309370 \\ https://hal.science/hal-00309370}

Submitted on 10 May 2010

HAL is a multi-disciplinary open access archive for the deposit and dissemination of scientific research documents, whether they are published or not. The documents may come from teaching and research institutions in France or abroad, or from public or private research centers.
L'archive ouverte pluridisciplinaire HAL, est destinée au dépôt et à la diffusion de documents scientifiques de niveau recherche, publiés ou non, émanant des établissements d'enseignement et de recherche français ou étrangers, des laboratoires publics ou privés. 


\title{
Physical Gels and Biopolymers
}

\author{
M. Rinaudo \\ CERMAV, CNRS - BP 68 \\ F-38042 Saint Martin d'Heres, France
}

We review some recent experimental results on physical gels made of biopolymers which are either vegetal (pectin, agarose) or animal (gelatin). These are either neutral (agarose) or electrically charged (pectin, gelatin). Two types of gels are considered :

- Thermoreversible gels, stabilized by secondary links and/or pseudo crystallization of multiple helices. There is usually hysteresis when cooling the system down or heating it up. For both gelatin and $\mathrm{K}$-carraghenan, it is shown that gelation occurs in two successive stages corresponding to two helicoidal distributions whose relative importance depends on $L$ ime.

- Ionic gels that are formed by selective fixing of bivalent ions such as calcium with the "egg-box" mechanism (pectin). The importance of the microstructure (a random sequential distribution of carboxylic groups) is analyzed. With light scattering and viscosity measurements we show the influence of charge density on gel formation.

\section{Introduction}

Nous souhaitons présenter un certain nombre de résultats expérimentaux rēcents sur des gels physiques formés par quelques biopolymères.

Les biopolymères sont certainement les premiers polymères connus pour former des gels physiques, c'est à dire des réseaux tridimensionnels non covalents avec, par exemple :

- les pectines ou l'agarose qui sont des polysaccharides gélifiants d'origine végétale

- ou la gélatine, protéine d'origine animale

Ces gêlifiants sont neutres du point de vue ionique (1'agarose) ou de nature polyêlectrolyte (pectine, gêlatine).

Ces polymères naturels sont d'abord stêréoréguliers ce qui va permettre d'obtenir la stabilisation d'une structure tridimensionnelle par un système coopératif de liaisons secondaires (liaisons hydrogène ou de Van der Waals) ou de liaisons ioniques.

Très généralement la transition de phase sol $\rightarrow$ gel des solutions aqueuses sera associée à la transition pelote + hêlice du biopolymère.

Nous nous proposons d'examiner deux types de gels : 
- les gels thermoréversibles stabilisēs par liaisons secondaires et/ou une pseudo-cristallisation d'hélices multiples ou de segments d'hélices associée au changement de phase. Le rôle de la qualité thermodynamique du solvant doit être
primordial.

- les gels ioniques pour lesquels la réticulation procède par fixation sélective d'ions divalents tels que le calcium.

\section{1 - Les gels thermorëversibles}

Nous prendrons comme exemple les gels de gélatine (collagène dénaturé) et de K-carraghēnane (polysaccharides d'algues marines) en essayant de mettre en évidence un certain nombre d'analogies dans le mécanisme de la transition sol $\rightarrow$ gel à partir des travaux récents de M. DJABOUROV et coll [1-3], J.Y. CHATELLIER [4-5], D. DURAND et coll [6-7] et de C. ROCHAS, M. RINAUDO [8-11] respectivement.

Les conditions opératoires sont cependant très différentes : 5 à $20 \%$ en poids pour les travaux sur la gélatine en solution dans $0,1 \mathrm{M} \mathrm{NaCl}$ ou dans 1 'eau ; 0,1 à $1 \%$ en poids pour le $K$-carraghénane à concentration variable en électrolyte.

\section{a) La gēlatine}

Le collagène est une protēine constituée par trois chaînes hélicoīdales associées en triple hélice ; dans les solutions aqueuses chauffées au dessus de $40^{\circ} \mathrm{C}$, il se produit un changement conformationnel de type hélice-pelote bien mis en évidence par la variation du pouvoir rotatoire $[\alpha](T)$. La position de la courbe dépend des vitesses de variation en température et les courbes obtenues en montée et descente de température présentent toujours une hystérésis.

Lorsque cette solution est ramenée à une température inférieure à la température de fusion conformationnelle du polymère $T_{M}$ (de $1^{\prime}$ ordre de $35^{\circ} \mathrm{C}$ ), on

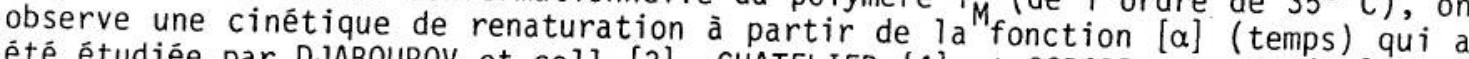
été étudiée par DJABOUROV et coll [3], CHATELIER [4] et GODARD et coll [12]. Les résultats expérimentaux sont analysés en proposant deux régimes cinétiques au moins :

- une cinétique d'évolution rapide de $[\alpha]$ donc du taux d'hêlicité qui correspond à la nucléation avec croissance fibrillaire (1'application de la théorie d'Avrami conduit à un indice $n=1$ ).

- une cinétique plus lente associèe à un accroissement de viscosité précédant le point de gel (4).

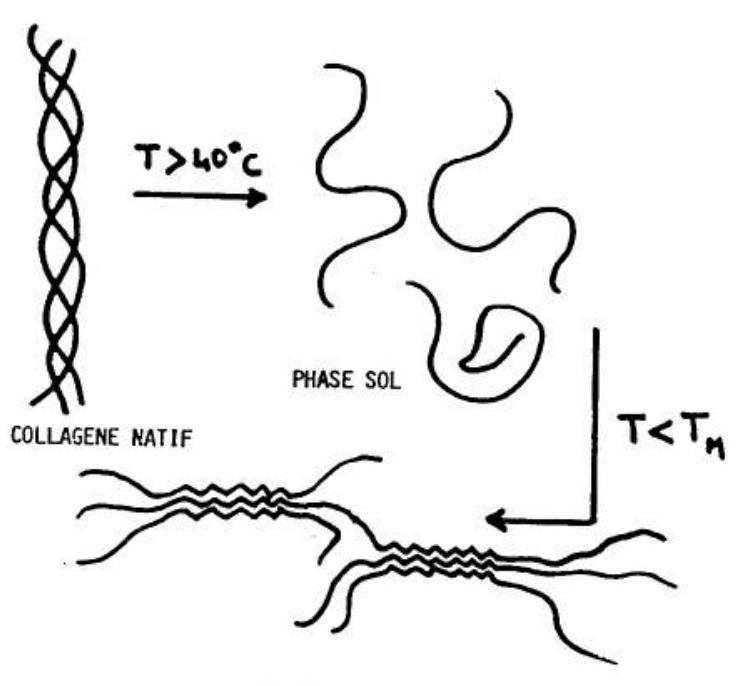

PHASE GEL - GELATINE

Figure 1 : Schêma de 1a gélification de la gélatine. 
D. DURAND et coll [6] montrent que le taux d'hểlicité $(h)$ au point de gel ne dépend que de la concentration en polymère soit : $14 \%(\mathrm{~h})$ et $7 \%$ (h) pour les concentration en polymère de $6 \%$ et de $11 \%$ respectivement; ils montrent de plus M. DJABOUROV [1] polymère placé pendante par ailleurs que ces gels sont hors d'équilibre ; un la fusion deux populations aux températures $22^{\circ} \mathrm{C}$ et $26,5^{\circ} \mathrm{C}$, présente à températures de fusion de $29^{\circ} \mathrm{C}, 36^{\circ} \mathrm{C}$ en hélices caractérisées par des haute température a la même caractérictivenent. Le pic tempērature de vieillissement domaines ordonnés augmente. Tous gel est proche de $T_{M}$ plus la stabilité des par au moins des segments de triples hêlices agrégées.

La formation du gel est contrôlée par la cinétique de renaturation des protéines; elle dépend de la concentration en gélatine et de la température adoptée. Le rôle du solvant n'est cependant jamais examiné.

\section{b) K-carraghénanes}

Avec ce polysaccharide, la cinétique de formation du gel n'est jamais dissociée de celle de la transition hêlice-pelote beaucoup plus rapide que celle

A faible concentration ionique en présence du cation $K^{+} \quad\left(c<C \quad 7.10^{-3}\right.$ équiv/l), on observe une transition thermique réversible hēlice-peloté en phase sol ; à partir de mesures thermodynamiques, on démontre que la conformation àdonnée est un dimère d'hélices [8]. Au dessus de cette concentration $C_{T}$, $C^{\prime}$ 'est à dire en réduisant la qualité thermodynamique du solvant, on obtient la voisinage de correspondant $\mathrm{a}^{\top}$ ' deux distributions un processus de gêlification en deux étapes relatives varient avec le temps de domaines hélicoïdaux dont les proportions est très analogue à celui de la gêlatine.

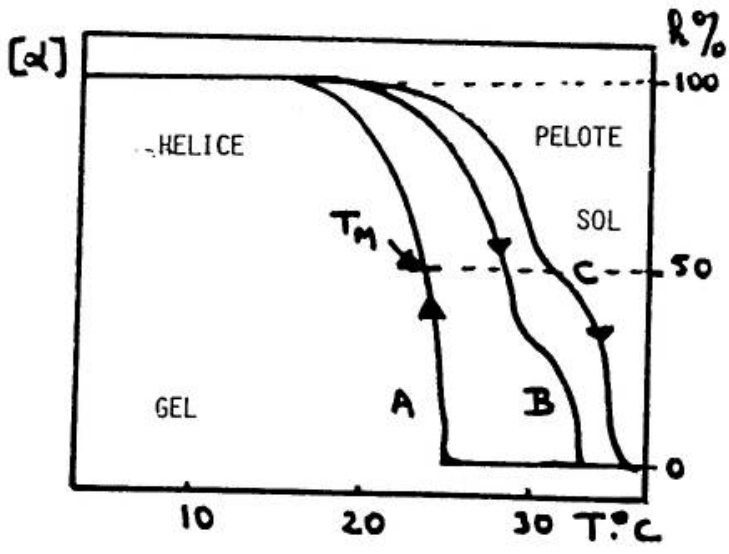

Figure 2: Influence de la température et du viellissement sur le pouvoir rotatoire et le taux d'hélicité (h\%) d'une solution de K-carraghénane. Courbes A et B : cycle thermique avec hystērésis (18 $\mathrm{C}$ /heure). Courbe C : viellissement $15 \mathrm{~h}$ à $21^{\circ} \mathrm{C}$.

Pour les carraghénanes nous exclúpons la nécessité de défauts de structure pour former un gel ainsi qu'un mécanisme impliquant un pont potassium tel que le
suggèrent MORRIS [13] et. SMIDSROD [14].

Nous proposons un mécanisme de réticulation par agrégation d'hélices (Fig. 3a) plutôt que par pontages par des segments de double hélice (Fig. 3b).

Parmi les gels thermoréversibles il faudrait enfin citer les gels mixtes obtenus en mélangeant des polysaccharides dont les structures moléculaires sont compatibles. C'est le cas du xanthane sous forme hélicoũdale et des zones lisses des galactomannanes. Il apparait que l'ensemble de ces systèmes implique des macromolécules de grandes anisotropies ayant un comportement de polymères 

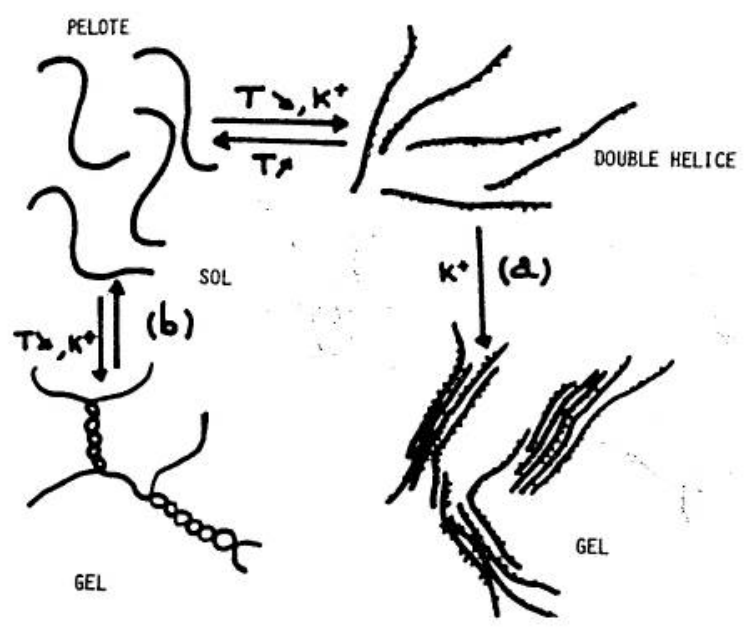

Figure 3 : Schéma de la

gélification du K-carraghénane.

a) par agrégats de doubles

hélices,

b) par zones hélicoīdales.

rigides. Leur comportement est cependant difficile à étudier en raison de la relation étroite entre les propriētés thermodynamiques et la transition conformationnelle qui se superpose au changement de phase. Cependant les comportements sont très proches de ceux obtenus sur le polyglutamate de benzyle, modèle de batonnet rigide dont le comportement est bien rendu par le traitement de Flory sur les cristaux liquides polymères [15]. Pour ce système, i] vient d'être proposé un modèle de réticulation donné à la figure 4 .

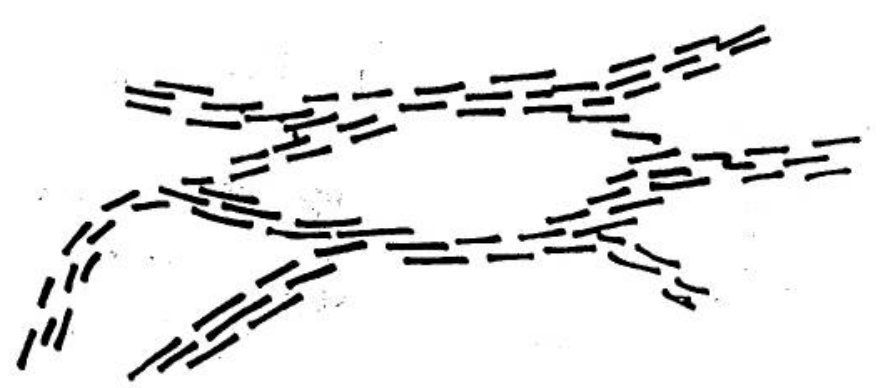

Figure 4 : Schéma de gélification d'un polymère rigide.

\section{2 - Les gels ioniques}

Les pectines faiblement méthylées (acide polygalacturonique à taux d'estérification variable) et les acides alginiques (copolymères d'acides mannuronique et guluronique) présentent de grandes analogies de structure moléculaire et forment des gels en présence d'ions calcium [16-17].

Il a été montré que dès que le degré de polymérisation était supérieur à 10 ou 15, il se formait des dimēres d'hêlices $2_{1}$ en présence de calcium dans le mécanisme dit de "boite à oeufs" (Fig. 5).

La fixation de calcium est spécifique et conduit avec le polymère à la formation de gel stable assimilable à un gel covalent; en revanche, l'ion magnésium ne forme ni dimères ni gels.

Nous avons également montré :

1) le rôle de la microstructure du polymère en comparant à même degré d'estérification un polymère partjellement hydrolysé par la soude (les groupes carboxyliques libres sont distribues au hasard sur la chaine) et par voie enzymatique (les sites carboxyliques sont en séquence) [17-18].

2) L'influence de la densité dé charge sur l'aptitude à former des gels. Les méthodes utilisées pour mettre en évidence l'agrégation sont des mesures de 1.'intensitê de lumière diffúsée et de viscosité.

Le comportement de ces gels ressémble à celui des gels covalents compte tenu de la durêe de viè grande des noeuds du réseau. 


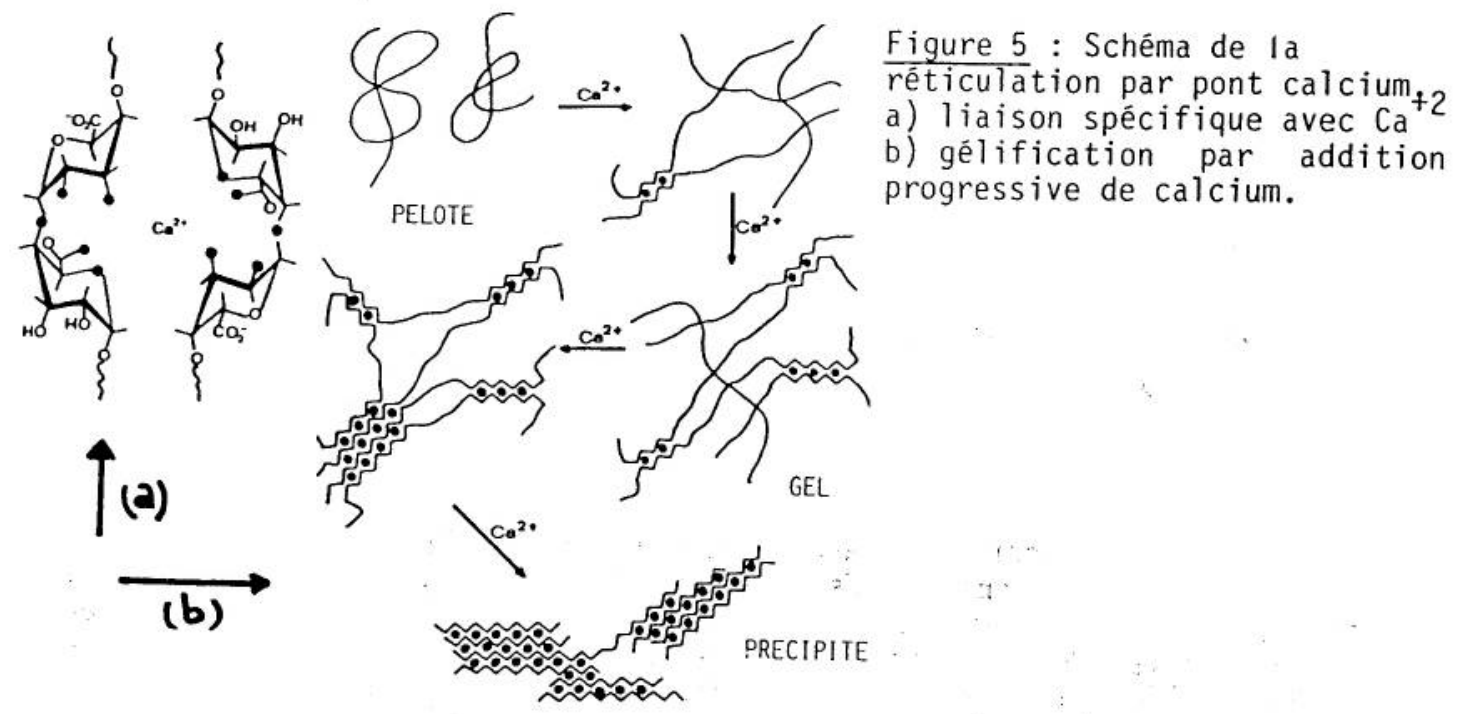

\section{CONCLUSION}

Il existe actuellement quelques biopolymères bien caractērisēs dont le comportement est bien maîtrisé et sur lesquels il serait intéressant de développer des études physiques.

De toutes facons, avec ces polymères il faudra être prudent dans le choix de l'échantillion et sa qualité ou son degré de pureté si on veut établir les lois de comportement. Il est en effet clair que des différences de microstructure des échantillons modifieront leur comportement physique. C'est ce que nous avons bien montré sur des pectines dont on avait contrôlé la distribution des sites ioniques.

\section{Références}

1 - M. DJABOUROV, P. PAPON : Polymer 24, 537-542 (1983).

2 - J. MAQUET, H. THEVENEAU, M. DJABOUROV, P. PAPON : Int. J. Biol. Macromol. 6 $162-163$ (1984).

3 - M. DJABOUROV, J. MAQUET, H. THEVENEAU, J. LEBLOND, P. PAPON : British Polymer J. (à paraître).

4 - J.Y. CHATELLIER : Thèse, Le Mans (France) (1983).

5 - J.Y. CHATELLIER, D. DURAND, J.R. EMERY : Int. J. Biol. Macromol. (sous presse).

6 - D, DURAND, J.R. EMERY, J.Y. CHATELLIER : Int. J. Biol. Macromol. (sous presse).

7 - J.R. EMERY, J.Y. CHATELLIER, D. DURAND : J. Physique (à paraître).

8 - C. ROCHAS : Thèse, Grenoble (France) (1982).

9 - C. ROCHAS, M. RINAUDO, Carbohydr. Res. 105 227-236 (1982).

10 - M. RINAUDO, C. ROCHAS, B. MICHELS : J. Chim. Phys. 80 305-308 (1983).

11 - C. ROCHAS, M. RINAUDO : Biopolymers 23 735-745 (1984).

12 - P. GODARD, J.J. BIEBUYCK, M. DAUMERIE, H. NAVEAU, J-P. MERCIER : J. Polym. Sci. Polym. Phys. Ed. $161817^{3}$ (1978).

13 - E.R. MORRIS, D.A. REES, C. ROBINSON : Mol. Biol. 138, 349 (1980).

14 - 0. SMIDSROD : I.U.P.A.C. 27 th International Congress of Pure and Applied Chemistry on charged polysaccharides. Pergamon Press (1980).

15 - P.J. FLORY, I. UEMATSU, Y. UEMATSU : in Liquid Cristal Polymers I (1-73). Advances in Polymer Science. Volume 59, Springer Verlag (1984).

16 - G. RAVANAT : Thèse, Grenoble (France) (1979).

17 - J. F. THIBAULT, M. RINAUDO : British Polymer J.' (sous presse).

18 - J.F. THIBAULT, M. RINAUDO : Biopolymers (sous presse). 